\title{
On the Querying for Places on the Mobile Web
}

\author{
Christian S. Jensen \\ Department of Computer Science, Aarhus University \\ Denmark \\ csj@cs.au.dk
}

The web is undergoing a fundamental transformation: it is becoming mobile and is acquiring a spatial dimension. Thus, the web is increasingly being used from mobile devices, notably smartphones, that can be geo-positioned using GPS or technologies that exploit wireless communication networks. In addition, web content is being geo-tagged. This transformation calls for new, spatio-textual query functionality. The research community is hard at work enabling efficient support for such functionality.

We refer to points of interest with a web presence as spatial web objects or simply as places. Such places thus have a location as well as a textual description. Spatio-textual queries return places that are near an argument location, typically the location of a user, and are relevant to a text argument, typically a search engine query. The talk covers recent results by the speaker and his colleagues aimed at providing efficient support for the spatio-textual querying of places. Specifically, the talk touches on questions such as the following:

- How to use user-generated content, specifically large collections of GPS records, for identifying semantically meaningful places?

- How to assign importance to places using GPS records and logs of queries for driving directions?

- How to retrieve the $k$ most relevant places according to both text relevancy and spatial proximity with respect to a query?

- How to support continuous queries that maintain up-to-date results as the users who issue queries move?

- How to take into account the presence of nearby places that are relevant to a query when determining the relevance of a place to a query?

\section{References}

1. Cao, X., Cong, G., Jensen, C.S.: Retrieving Top-K Prestige-Based Relevant Spatial Web Objects. PVLDB 3(1), 373-384 (2010)

2. Cao, X., Cong, G., Jensen, C.S.: Mining Significant Semantic Locations From GPS Data. PVLDB 3(1), 1009-1020 (2010)

3. Cong, G., Jensen, C.S., Wu, D.: Efficient Retrieval of the Top-k Most Relevant Spatial Web Objects. PVLDB 2(1), 337-348 (2009)

4. Venetis, P., Gonzales, H., Jensen, C.S., Halevy, A.: Hyper-Local, Directions-Based Ranking of Places. PVLDB 4(1) (2011) (to appear)

5. Wu, D., Yiu, M.L., Jensen, C.S., Cong, G.: Efficient Continuously Moving Top-K Spatial Keyword Query Processing. In: ICDE (2011) (to appear) 